\title{
La museología crítica y los estudios de público en los museos de arte contemporáneo: caso del museo de arte contemporáneo de Castilla y León, MUSAC
}

$M^{a}$ del Mar Flórez Crespo

Universidad de León

RESUMEN. La museología crítica surge de la crisis constante del concepto de museo como espacio de interacción entre el público y una colección. En este marco de revisión conceptual del museo y de la museología como disciplina científica, los centros y museos de arte contemporáneo han contribuido a avivar la controversia sobre la definición de museo y sus funciones. De manera pareja, algunos de ellos han experimentado una gran afluencia de público como es el caso del Museo de Arte Contemporáneo de Castilla y León (MUSAC). La evaluación de la interacción de su público y el museo es fundamental para entender este fenómeno y la función de este museo de arte contemporáneo.

Palabras clave: Museología crítica, estudios de público, Museo de Arte Contemporáneo de Castilla y León (MUSAC).

ABSTRACT. The critical museology arises from the constant crisis of the concept of museum as space of interaction between the public and the collection. In this frame of conceptual review of the museum and of the museology as scientific discipline, the centers and museums of contemporary art have helped to intensify the controversy on the definition of museum and its functions. Some of them have experienced a public's great abundance since it is the case of the Museum of Contemporary Art of Castilla and León (MUSAC). The evaluation of the interaction of its public and the museum is fundamental to understand this phenomenon and the function of this museum of contemporary art.

Key Words: Critical museology, public evaluation, Museum of Contemporary Art of Castilla and León (MUSAC).

\section{LA MUSEOLOGÍA CRÍTICA Y LOS MU- SEOS DE ARTE CONTEMPORÁNEO}

A lo largo del siglo XX fueron aparecido diferentes escuelas museológicas con diversas tendencias. La "nueva museología", que surge como contraposición a la "museología tradicional", es quizás la que más relevancia ha tenido hasta el presente. ${ }^{1}$ En esta línea y en los últimos años se ha desarrollado una nueva corriente de pensamiento que busca dar respuesta al papel de los museos en el siglo XXI: es la denominada "museología crítica".

La actualidad de este fenómeno museológico es evidente. En fechas recientes han

-

\footnotetext{
${ }^{1}$ Una visión general se puede obtener en L. A. ALONSO FERNÁNDEZ, Introducción a la nueva museología, Madrid, 1999.
}

aparecido dos obras que reconocen de manera plena esta rama de la ciencia de la museología. Una de ellas es Planteamientos teóricos de la museología, en cuyo capítulo quinto la autora, Francisca Hernández, trata ampliamente las tendencias del pensamiento museológico y en general del apartado teórico de esta disciplina, concretando el caso singular de la museología crítica. La otra se dedica completamente a su análisis, incluso recibe tal título, Museología crítica, y ha sido realizado por Joan Santacana Mestre, y Francisco Xavier Hernández Cardona. ${ }^{2}$ Sin embargo, ya en 2003 Jesús Pedro Lo-

2 F. HERnÁNDEZ HeRnÁNDEZ, Planteamientos teóricos de la museología, Gijón, 2006, en concreto pp. 200-226. J. Santacana Mestre, y F. X. Hernández Cardona, Museología crítica, Gijón, 2006, de esta última obra se recomienda en especial los apartados dedicados a "La 
rente Lorente y David Almazán planteaban el debate en una recopilación de artículos de diversos museólogos e historiadores del arte españoles que versaban sobre la singularidad de la aplicación de la museologia crítica al arte contemporáneo, y sus museos o centros. ${ }^{3}$

La denominación procede del ámbito de la investigación universitaria, en concreto, del ámbito de la Historia del Arte. En sus planteamientos se cuestiona el papel del museo en la actualidad, "dentro de un contexto de revisionismo de la post modernidad en que la cultura dominante ha sido visibilizada, desmitificada $\mathrm{y}$ desestructurada."

Así, la museología crítica surge de la crisis constante del concepto de museo como espacio de interacción entre el público y una colección, y como consecuencia de una política cultural. Esta vertiente adquiere su punto álgido en el debate dentro de la polémica de esos museos y/o centros de arte contemporáneo. En este marco de revisión del concepto de museo y de la museología como disciplina científica, dichos centros y museos de arte contemporáneo han contribuido a avivar la controversia sobre la definición de museo y sus funciones, dentro de la disciplina de los museos de arte. ${ }^{5}$

La "museología crítica", en palabras de María Teresa Martín, es "una revisión a esa nueva museología de los años setenta del XX que hablaba de la muerte del museo de arte y de la superación de esta institución tradicional". ${ }^{6}$ Según Carla Padró, es una forma de reflexionar sobre los museos como zonas de conflicto e intercambio, parafraseando a L. Bunch, o J. Clifford cuando se refiere al museo como "zona de confluencia y de contacto". 7 Pero además, en su visión de la museología crítica, dicha autora

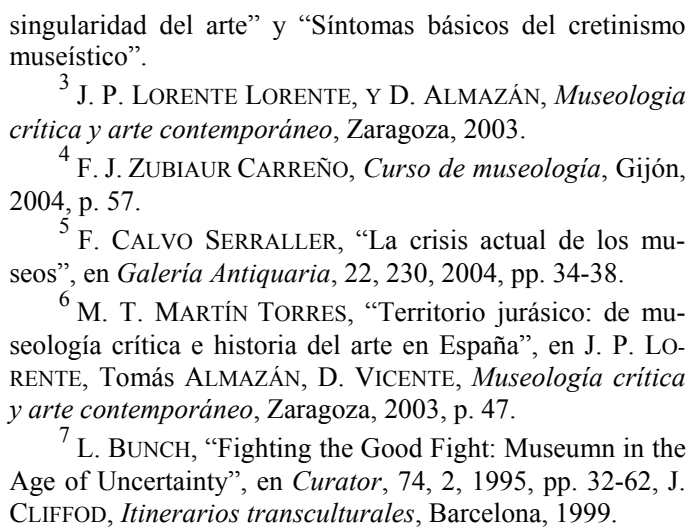

${ }^{4}$ F. J. ZuBiaur CARreño, Curso de museología, Gijón, 2004, p. 57.

5 F. Calvo Serraller, "La crisis actual de los museos”, en Galería Antiquaria, 22, 230, 2004, pp. 34-38.

${ }^{6}$ M. T. MARTín TORRES, "Territorio jurásico: de museología crítica e historia del arte en España”, en J. P. LORENTE, Tomás AlmazÁn, D. ViCENTE, Museología crítica y arte contemporáneo, Zaragoza, 2003, p. 47.

${ }^{7}$ L. BunCH, "Fighting the Good Fight: Museumn in the Age of Uncertainty", en Curator, 74, 2, 1995, pp. 32-62, J. CLIFFOD, Itinerarios transculturales, Barcelona, 1999.

enfatiza el carácter interpretativo de los museos y de cómo su discurso se transcribe en diferentes tipos de políticas culturales, o de maneras a través de las cuales se han construido y entendido los museos. Ante esas posibles maneras, la que nos ocupa se enmarca dentro de la que entiende el museo como "lugar de duda, de pregunta, de controversia y de democracia cultural... Estaríamos ante una cultura postmoderna revisionista, donde se considera que para entender las formas del conocimiento y de comprensión se debe partir de la noción de lucha y de conflicto". El museo es entendido como comunidad de aprendizaje, pero resultado de un proceso de negociación entre diferentes poderes (consorcios, comités de expertos, artistas, visitantes, comunidades, etc.) que son los que van a definir la política museal. ${ }^{8}$

En un marco más amplio, esta línea de reflexión de la museología, encaja también dentro de la corriente de la pedagogía crítica. Así, el museo como espacio de enseñanza no formal, es el lugar idóneo donde se crea una comunidad de aprendizaje mediante la negociación y dialogo con el público y en torno a las exposiciones de los museos. Esta postura replantea la función de investigación de museos como herramienta desde la educación. El paso que se produce supera el paradigma comunicativo del museo de la nueva museología hacia uno de negociación y participación cultural propio de la museología crítica. ${ }^{9}$

En resumen, la corriente de la museología crítica busca formar a una ciudadanía más abierta a expresar su opinión, no sólo consumista. Los museos, y en especial en los recientes museos de arte contemporáneo, poseen obras que se están creando en la actualidad, por ello se plantean como lugar idóneo para el diálogo y también como razón del debate. El público se asume desde una posición reflexiva y emancipadora de un pasado donde, hasta ahora, dicho público se limitaba a aceptar lo que se le decía

-

${ }^{8}$ C. PADRÓ, "Museología crítica como forma de reflexionar sobre los museos como zonas de conflicto e intercambio", en J. P. LORENTE, Tomás ALMAZÁn, D. ViCENTE, Museología crítica y arte contemporáneo, Zaragoza, 2003, pp. 57-60.

${ }^{9}$ Es de interés la obra de A. AuBERT SIMÓ (Et. al.), Dialogar y transformar: pedagogía crítica del siglo XXI, Barcelona, 2004, y sobre todo la obra reciente de J. RODRIGo Montero, Pedagogía crítica y educación en museos. Marcos para una educación artística desde las comunidades, Valladolid, 2006. 
qué era arte y qué no. No cabe duda que los museos o centros de arte contemporáneo se tienen que hacer cargo del riesgo que ello implica, pero, a la vez, aceptar el avance en la consecución de sus resultados.

LOS MUSEOS DE ARTE CONTEMPORÁNEO: EL MUSEO DE ARTE CONTEMPORÁNEO DE CASTILLA Y LEÓN (MUSAC)

El fenómeno de la creación de museos de arte contemporáneo en las últimas décadas del siglo XX hasta la actualidad es un hecho evidente. Desde el momento en que en España se empieza a tomar conciencia de la importancia del arte contemporáneo con la creación del Museo Español de Arte Contemporáneo, MEAC hoy Museo Nacional Centro de Arte Reina Sofía (MNCARS) pasando por el fenómeno Guggenheim Bilbao, la carrera por la apertura de este tipo de instituciones ha sido espectacular. ${ }^{10}$ Muchos de ellos han sido creados por mera competencia política o fruto del activismo cultural actual, sin pensar en su línea de actuación e implementación. Sin entrar en el debate que se ha planteado sobre la pertinencia o no de denominar a dichos espacios museos o centros, no cabe duda de que la afluencia de público a los mismos ha sido otra de las singularidades que ha acompañado de manera pareja a su creación. En este discurso es enmarca el Museo de Arte Contemporáneo de Castilla y León (MUSAC), inaugurado en abril de 2005, que se analiza a continuación.

El origen del MUSAC se encuentra en la intención de la Junta de Castilla y León de crear un centro de arte contemporáneo para la comunidad. El antecedente legal del museo se establece en la Ley 10/1994, de 8 de julio, de Museos de Castilla y León. Así pues, en esos momentos de la década de los noventa del siglo XX, la Administración regional se comprometía a crear un centro dedicado al arte contemporá-

${ }^{10}$ En torno a dicho fenómeno y a sus antecedentes históricos se puede consultar F. MARTín MARTín, "Panorama de los centros de arte contemporáneo en España: proyectos y realidades", en J. P. LORENTE, Tomás ALMAZÁN, D. VICENTE, Ob. cit, pp. 293-315, y sobre todo, M. A. LAYUNO RosAS, Museos de arte contemporáneo en España: del "Palacio de las Artes" a la arquitectura como arte, Gijón, 2004. neo en su ámbito administrativo autonómico, sin definir aún dónde o cómo. ${ }^{11}$

Casi diez años después su creación el proyecto toma un nuevo impulso. En el punto tercero del Plan de Actuación en Museos de Castilla y León, el ente regional se comprometió a poner en funcionamiento los servicios correspondientes al Centro de Arte Contemporáneo de Castilla y León. ${ }^{12}$ El emplazamiento se había decidido en la propia ciudad de León a pesar de las críticas y controversia creada en el momento. ${ }^{13}$

El primer proyecto se diseñó en el año 1999 dentro del "Concurso de ideas para el Auditorio de León" convocado por el ayuntamiento de la ciudad que, con posterioridad, y por acuerdo entre la Junta de Castilla y León y dicho municipio, se transforma en el Centro de Arte Moderno y Contemporáneo de Castilla y León. Sin embargo, se vio más oportuno realizar un nuevo proyecto en un solar más espacioso de la ciudad. Los arquitectos en quienes recayó el proyecto arquitectónico fueron Luis Moreno Mansilla y Emilio Tuñón. ${ }^{14}$

-

${ }^{11}$ En su artículo 13, "Centro para el arte contemporáneo", dice: "1. La Administración de la Comunidad Autónoma de Castilla y León creará un centro dedicado a la documentación, difusión, exhibición y fomento del arte contemporáneo en Castilla y León, que coordinará asimismo las iniciativas museísticas relacionadas con este campo en el ámbito de la Comunidad Autónoma. 2. Su estructura y funcionamiento se desarrollarán reglamentariamente. Ley 10/1994, de 8 de julio, de Museos de Castilla y León.

12 “... previa dotación de los recursos y disposiciones que sean necesarios para establecer la estructura y funciones durante el período de vigencia del mencionado Plan de Actuación". Acuerdo 64/2003, de 15 de mayo, de la Junta de Castilla y León, por el que se aprueba el Plan de Actuación en Museos de Castilla y León para el período 20032008.

${ }^{13}$ En aquellos años en los que se había aprobado recientemente la Ley de Museos de Castilla y León, y se debatía sobre los posibles emplazamientos del "centro de arte contemporáneo" de la comunidad, se planteaban como posibles León, Zamora y Salamanca, siendo definitivo el emplazamiento de León. Hubo posturas que defendieron las candidaturas de Zamora, e incluso la de Valladolid, antes que la seleccionada, aunque el lugar recayó en la capital leonesa. J. GonZÁlez PoSADA, "La vida cultural", en A. GARCÍA Simón, J. ORTEGA VALCÁRCEL (eds.), Historia de una cultura: Castilla y León/Informe, vol. 4, Valladolid, 1996, p. 436

${ }^{14}$ E. Tuñón y L. Moreno Mansilla, "Centro de Arte Moderno y Contemporáneo de Castilla y León", en Revista de Museología, 17, 1999, pp. 90-95. También se puede consultar Concurso de ideas para el Auditorio Ciudad de León, León, 1995, J. L. DE LAS RIVAS SANZ, “Arquitectura 
$\mathrm{Su}$ ámbito de actuación corresponde a todo el territorio de la Comunidad Autónoma y de entre las funciones y competencias asignadas al Museo se concede especial importancia a los aspectos relativos a la documentación, exhibición y fomento del arte actual, así como a la difusión y conocimiento del mismo. En esta labor la intervención de diferentes entidades y profesionales debe ser fundamental, razón por la que se incluye entre las actuaciones del Museo el establecimiento de relaciones de cooperación y colaboración con otros centros culturales y de investigación para la realización de proyectos y actividades, y la coordinación de iniciativas museísticas en su ámbito de actuación. ${ }^{15}$

Las actividades destinadas al público tanto general como especializado, cobran gran importancia. La programación de proyectos de investigación, de actividades didácticas, o de exposiciones temporales, tienen especial protagonismo en la actividad y servicios de este museo y se constituyen en uno de los ejes fundamentales para su programación. ${ }^{16}$

en Castilla y León", en A. GARCía SimÓN, J. ORTEGA VAlCÁRCEl (eds.), Historia de una cultura: Castilla y León/Informe, vol. 4, Valladolid, 1996, p. 593.

${ }^{15}$ Todo ello viene definido por lo estipulado en la Ley del Patrimonio Histórico Español de 1985, en los reglamentos que la desarrollan, y en las normas que afectan al los museos y a la protección del patrimonio en Castilla y León: Ley 12/2002, de 11 de julio, de Patrimonio Cultural de Castilla y León y disposiciones que la desarrollan, además de la Ley 10/1994, de 8 de julio, de Museos de Castilla y León, y su reglamento y demás disposiciones de desarrollo.

${ }^{16}$ Según su decreto de creación, artículo 6: "Corresponden al Museo de Arte Contemporáneo de Castilla y León las siguientes funciones: 1. La conservación, documentación, difusión, exhibición y fomento de la creación de Arte Contemporáneo, en cualquiera de sus manifestaciones, en el ámbito de la Comunidad Autónoma de Castilla y León. 2. La coordinación de las iniciativas museísticas relacionadas con el Arte Contemporáneo en la Comunidad Autónoma de Castilla y León. 3. Exhibir sus colecciones para mostrar y explicar el Arte Contemporáneo en todos sus aspectos. 4. Organizar proyectos y exposiciones temporales que muestren las manifestaciones del arte actual en sus diferentes ámbitos territoriales y temporales. 5. Desarrollar actividades didácticas en relación con los contenidos y objetivos del Museo. 6. Elaborar, editar y publicar, en cualquier tipo de soporte, catálogos, guías, estudios, monografías, documentales, grabaciones, o cualquier otra obra relacionada con las funciones y objetivos del Museo. 7. Impulsar y promover los estudios y el conocimiento del arte actual y fomentar su análisis cultural y social. 8. Facilitar el estudio de sus fondos a investigadores y profesionales, sin perjuicio de las restricciones que por razón de la conservación de los bienes puedan establecerse. 9. Establecer relaciones de cooperación y colaboración con otros museos, universidades, centros de investigación o instituciones culturales nacionales o extran-
Además, el museo nace con el interés de modificar el concepto del arte en el siglo XXI, por ello, las colecciones y fondos del MUSAC agrupan los movimientos artísticos internacionales del arte actual, realizados sobre cualquier soporte y mediante cualquier técnica. En términos generales es el primer museo en el mundo que incorpora todo tipo de artes tecnológicos y digitales de plena y absoluta actualidad. La colección, por tanto, está en continuo crecimiento. Con este museo se pretende también contribuir a la coordinación de las actuaciones sobre las manifestaciones artísticas actuales en Castilla y León.

La compra de piezas ha sido una de las actividades fundamentales que se ha llevado a cabo en los años previos a la apertura del museo y sigue siéndolo en la actualidad. Sus fondos recogen los movimientos artísticos internacionales del arte actual, realizados sobre cualquier soporte y mediante cualquier técnica. En él se incorporan todo tipo de artes tecnológicos y digitales. Dado que aún está en fase de adquisición de obras, sus fondos están en continuo crecimiento. Para su correcto control ha sido fundamental la labor de registro y documentación de las mismas. El registro, inventario y catalogación de los bienes adscritos al Museo se realiza de acuerdo con la normativa específica establecida al respecto en las leyes apuntadas.

\section{Fondos, colecciones y exposiciones}

Según el Plan de Museos autonómico (2003 / 2008) el Centro de Arte Contemporáneo de Castilla y León tenía que asumir un papel primordial en el estímulo a la creación artística en la Comunidad, potenciando las diferentes manifestaciones artísticas de la época actual y facilitando su comprensión y disfrute. Así el espíritu común de los fondos iniciales requería obras realizadas, como se ha dicho, sobre cualquier soporte y mediante cualquier técnica dentro de la creación artística contemporánea. La cronología de esa contemporaneidad se ha concretado en la etapa que se desarrolla durante el

jeros, para el intercambio de experiencias y conocimientos, así como para la organización de proyectos o actividades conjuntas. 10. Cualesquiera otras que sobre el Arte Contemporáneo le encomiende la Junta de Castilla y León.". Decreto 24/2005, de 22 de marzo, por el que se crea el Museo de Arte Contemporáneo de Castilla y León. 
último decenio del siglo $\mathrm{XX}$ y el primer decenio del siglo XXI. ${ }^{17}$

La labor de selección y recopilación se ha encargado a un grupo de especialistas de reconocido prestigio que han trabajado en ello durante los años precedentes a su inauguración. Todas las piezas que se han ido asignando al museo han sido adquiridas por la Junta de Castilla y León en los últimos años según el asesoramiento científico de dicho equipo compuesto por Jesús María Gómez Sanz, Director, Fundación Siglo para las Artes en Castilla y León; Rafael Doctor Roncero, Director del MUSAC y Director de Artes Plásticas de la Fundación Siglo; Agustín Pérez Rubio, Conservador Jefe del MUSAC; Octavio Zaya, Crítico y Comisario Independiente, Co-comisario, Documenta XI; José Guirao, Director, Casa Encendida, Madrid; Estrella de Diego, Crítica de Arte Independiente y Profesora de Historia del Arte, Universidad Complutense de Madrid; Javier Hernando, Crítico de Arte Independiente y Profesor de Historia del Arte, de la Universidad de León, y María Jesús Miján, Coordinadora de Proyectos Expositivos de la Junta de Castilla y León. ${ }^{18}$

Por todo ello, la colección aún está configurándose en lo que hoy es, en palabras de su director, "Museo de la actualidad" hacia lo que será el "Museo del cambio de milenio". Se presentará de manera definitiva alrededor de 2010. Abarcaría así una cronología de 20 años, aproximadamente los diez años anteriores y posteriores al cambio de dicho milenio. Esa

-

${ }^{17}$ El Plan de Actuación de Museos promueve la renovación e impulso de los museos regionales "atendiendo a criterios de calidad, diversidad, complementariedad y competencia". Acuerdo 64/2003, de 15 de mayo, de la Junta de Castilla y León, por el que se aprueba el Plan de Actuación en Museos de Castilla y León. (B.O.C. y L. n. ${ }^{\circ}$ 95, de 21 de mayo).

${ }^{18}$ Las características principales de su colección abordan los siguientes aspectos: el diálogo entre el arte producido en España y el que se produce en el resto del mundo dentro del mismo ámbito de lo artístico, el intento de búsqueda de obras significativas de la producción de los artistas representados en la colección, la convivencia de técnicas tradicionales y contemporáneas, y la interconexión entre artistas de reconocida trayectoria con otros casi noveles. La clasificación inicial de sus fondos se ha dividido en seis secciones que, sin pretender una división dogmática, sirve para dar forma y mejor comprensión a la totalidad de sus fondos. Son: Identidad individual, Identidad Social; Identidad Cultural; Mirada externa: El otro; Mirada externa: Paisaje, y Mirada Poética. Más datos sobre los artistas que lo componen y el espíritu de la colección se pueden consultar en AA. VV., MUSAC. Colección, vol. I, 2005. cronología será la que enmarque las obras que formarán la base definitiva de la colección del MUSAC sin que ello signifique que la recopilación de obra se estanque ahí. Se podrá incrementar con los bienes que sean asignados al Museo de acuerdo con las diferentes modalidades legalmente admitidas (donaciones, legados, compras, premios...) para redundar en el enriquecimiento de sus fondos, además de ser depósito de otros bienes según sea acordado por la Consejería autonómica competente en materia de cultura.

Respecto de su sistema de exposiciones, la programación general del Museo está concebida para su renovación periódica. Desde su inauguración hasta el presente lleva realizadas 5 muestras, que son: ${ }^{19}$

- Emergencias: Exposición inaugural colectiva, tuvo lugar del 1 de abril al 21 de agosto 2005. Se planteó como una reflexión sobre la actualidad desde un punto de vista político y social y buscando la concienciación colectiva de la problemática del ser humano en el mundo a través de distintos proyectos creados por artistas actuales.

- Dora García: Vibraciones, Shirin Neshat: La última palabra y Sujeto: Exposición triple que tuvo lugar del 10 de septiembre al 6 de diciembre 2005. El proyecto de Dora García reflexionaba sobre el sonido y la música. Shirin Neshat tenía recogidas en esta exposición varias piezas representativas de su obra desarrollada en los últimos 10 años, 20 fotografías y 6 vídeo instalaciones, de entre las 11 producidas por la artista iraní. Sujeto consistió en una compilación razonada de retratos.

- Enrique Marty. Flaschengeist. La caseta del alemán, Pröblemäs büenös. 4 obras de Pipilotti Rist y amigäs, y Fusion: Exposición triple que tuvo lugar del $17 \mathrm{de}$ diciembre de 2005 al 16 de abril de 2006 que es la que se evalúa en este proyecto.

- Globos Sonda: Exposición colectiva inaugurada el 6 de mayo y presente hasta

-

${ }^{19} \mathrm{La}$ información sobre el programa de exposiciones pasadas, actuales, y futuras se puede consultar en: www.musac.org.es 
el 10 de septiembre de 2006. Se anuncia con carácter de continuidad, como bienal, para ser testimonio de la labor de diferentes artistas y creadores, individuales o colectivos, en su mayoría noveles.

En la actualidad se está llevando a cabo el último proyecto que consta de 4 muestras dedicadas a otros tantos artistas actuales:

- Muntean \& Rosenblum, Julie Mehretu, Felicidad Moreno, Daniel Verbis: Exposición cuádruple, inaugurada el 23 septiembre 2006 y presente en el MUSAC hasta el 7 enero 2007. La primera denominada "Make death listen (Haz que la muerte escuche)", es una selección retrospectiva de piezas de la pareja artística compuesta por Markus Muntean y Adi Rosenblum. Al igual que el caso anterior, "Black City (Ciudad Negra)", titula la primera exposición individual en España -y también en Europa- de Julie Mehretu. La instalación diseñada para el museo de Daniel G. Verbis, "misojosentusojosderamándose", recrea su singular universo creativo a través de la pintura y la experimentación con diversos materiales. Por último "hipnÓptico" es el título con el que se presentan más de una docena de piezas de la artista Felicidad Moreno y una instalación que ha creado con carácter específico para el MUSAC.

Hasta la actualidad, todas ellas han sido gratuitas para fomentar el acceso libre de todo el público que desee conocer el museo y las propuestas expositivas de su equipo. Además se llevan a cabo otro tipo de proyectos expositivos que se enmarcan dentro de la programación habitual del museo con una actividad singular. Son las exposiciones que tienen lugar en el "Laboratorio 987" y en el "Proyecto Vitrinas".

El Laboratorio 987, es la sala de proyectos artísticos específicos del MUSAC y se presenta como un espacio anexo que funciona independientemente a la programación general del mismo, con una periodicidad de renovación de unos 2, 3 meses. En él han participado: Silvia Prada (1 de abril - 5 de junio de 2005), Fikret Atay (22 de junio - 21 de agosto de 2005), Abigail Lazkoz (10 de septiembre - 16 de octubre de 2005), Ryan McGinley (Del 28 de octubre 6 de diciembre), Wilfredo Prieto (Del 17 Diciembre 05 - 19 Febrero 06), y Monika Sos- nowska (Del 4 de marzo al 16 de abril de 2006), etc. $^{20}$

El Proyecto Vitrinas también funciona de manera paralela a la programación del resto de espacios expositivos. Se desarrolla en un ámbito singular del hall de entrada compuesto por dos grandes vitrinas de cristal, a modo de escaparate. Este espacio acoge propuestas próximas al mundo de la moda, de la música, del diseño y de otras industrias y artes que se circunscriben dentro de la producción actual contemporánea. En ella han participado artistas como Daniel G. Verbis (1 de abril - 21 de agosto de 2005), Locking Shocking (10 de septiembre - 4 de diciembre 2005), Vasava (17 de Diciembre 2005 - 16 de Abril 2006) y, desde el 6 de mayo hasta el presente de esta publicación, Funky Projects. Ante tal dinamismo expositivo, hace interesante una labor constante de evaluación de sus diferentes dimensiones en relación con el público.

En resumen, el Museo encaja en los principios en los que se mueve la museología crítica. Se construye con el propósito de ser un lugar interrelacional donde el público deje de ser un mero elemento pasivo que contempla y a su vez participe y sea informado de ese proceso de configuración de sus fondos. Talleres, charlas, encuentros con los artistas, guías didácticas y obras en las que el espectador interactúa, son algunos de los elementos imprescindibles en la dinámica del MUSAC.

\section{LA MUSEOLOGÍA CRÍTICA Y LOS ESTU- DIOS DE PÚBLICO EN LOS MUSEOS DE ARTE CONTEMPORÁNEO}

Los estudios de público en el museo pueden tener varias orientaciones. Una clasificación de las principales podría ser la siguiente:

- Marketing y estudios de mercado: es el uso más frecuente, parten de la metodología de trabajo de estudios habituales de análisis del consumo turístico, adecuados para el diseño de estrategias de atracción y fidelización de público. En los museos se enmarcan dentro del departamento de difusión y comunicación y en los últimos años han aportado muy buenos resultados de atracción de público. Sin embargo

\footnotetext{
${ }^{20}$ En el momento en el que se publica este artículo se expone, "Exvoto. Where is Nikki Black?" título que da nombre a la muestra que Philipp Fröhlich ha creado para el museo.
} 
la masificación de visitantes y la banalización del arte ha sido una de sus principales consecuencias. ${ }^{21}$ También se enmarcan en este contexto la evaluación de opinión o sondeos sobre intereses de consumo, relacionado con estudios de marketing. Se elaboran para la mejora de actividades y servicios del museo. ${ }^{22}$

- Montaje exposiciones. Es una rama de investigación del museo que tiene su origen en Estados Unidos. Los tipos de evaluación aplicables a este fin son variados. El más frecuente es la observación del público. $^{23}$

- Evaluación del aprendizaje y formación del visitante (captación del mensaje, por cualquier categoría de visitante, con edades, intereses, motivaciones o nivel económico, etc., diferentes). ${ }^{24}$

Cualquiera de estas orientaciones concilia los estudios de público y la corriente de la museología crítica ya que ésta se encontraría reflejada en su meta por todas ellas al buscar los objetivos generales evaluables en cada una. Sin embargo, donde mejor encaja en cuanto a objetivos concretos es en la última opción, aunque con ciertos matices. En el caso de la evaluación de la formación del visitante no se trata tanto de

${ }^{21}$ K. MOORE, La gestión del museo, Gijón, 1998, y N. KOTLER y P. KotLER, Estrategias y marketing de museos, Barcelona, 2001. Ambas obras plantean los estudios de público como herramienta de marketing para museos enfocado hacia la formación de una audiencia que consolide una base amplia de visitantes, y que dicho museo estreche relaciones con el público asiduo. Según esta orientación, la manera en que el museo establece y controla su relación con el público tendrá como consecuencia la mejora permanente de sus servicios y el nacimiento, afianzamiento o proyección de la imagen del mismo.

${ }^{22}$ En general, M. C. VALDÉS SAGÜES, La difusión cultural en el museo: servicios destinados al gran público, Gijón, 1999.

${ }^{23}$ Ejemplos interesantes son M. ASENSIO, E. POL y M. Gomis, Planificación en museología: el caso del Museu Marítim de Barcelona, Barcelona, 2001, o la propuesta que hace A. GaRCía BlanCo, La exposición: un medio de comunicación, Madrid, 1999. Sobre los tipos de evaluación, es fundamental M. E. PÉREZ SANTOS, Estudio de visitantes en museos: metodología y aplicaciones, Gijón, 2000. También se puede consultar lo que afecta a este apartado en M. ASENSIO y E. POL, "Evaluación de exposiciones", en J Santacana Mestre y N. Serrat Antolí (Coords.), Museografia didáctica, Barcelona, 2005.

${ }^{24}$ M. ASENSIO y E. POL, Nuevos escenarios de educación. Aprendizaje informal sobre el patrimonio, los museos y la ciudad, Buenos Aires, 2002. la valoración del aprendizaje sino del modo de interacción con el visitante en la construcción del museo; trata la evaluación en la formación del gusto a través de la participación del visitante en la labor de la institución. Ésta se lleva a cabo con actividades, talleres, información pública sobre la compra de obra, sobre las propuestas expositivas... El binomio de discusión que podría denominarse "Arte actual - público actual" tiene su marco idóneo en el espacio del museo de arte contemporáneo. Es el lugar donde se produce el "feedback", la retroalimentación del mensaje que intercambia el museo con el público $\mathrm{y}$, a su vez, el público con el museo. ${ }^{25}$

Éste es un matiz importante en la orientación de estos estudios que encaja con el espíritu de los museos de arte contemporáneo: a través de estos análisis se puede valorar la realidad de ese intercambio en constante proceso de creación. El museo es el puente e intérprete entre el artista y el público, y en los tres casos son coetáneos, viven la misma realidad cronológica.

\section{PROYECTO DE ESTUDIO DE PÚBLICO LLEVADO A CABO EN EL MUSAC}

\section{Marco teórico}

El MUSAC lleva a cabo la evaluación de su público a través del Departamento de Educación y Acción Cultural, DEAC. Éste realiza los estudios de evaluación tanto de las actividades que propone como del público que accede al museo. Respecto de este último lo hace desde una posición únicamente cuantitativa. Se toman datos sobre el número de visitantes que reciben al día en horario de mañana y tarde, además de preguntar su procedencia.

A partir de esta información se elaboran memorias sumativas de los talleres y servicio de visita guiada, de uso interno del museo y en concreto del DEAC. Además, la información sobre la cantidad de público visitante se emplea también por el Departamento de Comunicación.

\footnotetext{
${ }^{25}$ Se recomienda la interesante reflexión que al respecto realizan I. DíAZ BALERDi y A. UNZU IRAOLA, "La mirada que construye. Competencias y extravíos", en J. P. LORENTE, Tomás AlmaZÁn, D. ViCENTE, Ob. cit, pp.185-201, y en concreto, lo que comentan en las pp. 195-199, en lo que afecta a la relevancia de los estudios de público en la evaluación de la consecución de objetivos, y en especial, según expresan dichos autores ya en el propio título, de evitar "extravíos" en la política museográfica de museos y centros de arte contemporáneo.
} 
El elevado número de visitas que está recibiendo es reflejo del impacto de su labor, y además suele ser un frecuente motivo de aparición del museo en prensa.

Dada esta situación se hace interesante tratar la evaluación de otros campos que son útiles para ayudar a entender el fenómeno de tal afluencia de visitantes, su comportamiento o reacción ante las exposiciones y un perfil más definido del público, de sus hábitos, del uso de los servicios y actividades del museo o de su opinión sobre el mismo y la colección.

\section{Especificación de la demanda}

El MUSAC es una institución centrada en la colección, conservación y difusión del arte contemporáneo más actual. Como ya se comentó, lleva apenas un año de andadura y en su primera etapa aún sigue recopilando obra y manteniendo una política de exposiciones únicamente temporales. En esas exposiciones se exhiben sus fondos además de piezas prestadas o hechas ex profeso por un artista para la muestra en cuestión. De la misma manera, mantiene la gratuidad del acceso aunque está previsto cambiar esta dinámica.

Respecto a sus instalaciones, la superficie total de exposición es de $3.400 \mathrm{~m}^{2}$, algo más de un tercio del total de la superficie construida $-9.700 \mathrm{~m}^{2}$ - y la mitad de los metros cuadrados útiles en planta baja $-7.850 \mathrm{~m}^{2}$ - De entre sus áreas de uso público destacan la biblioteca $\mathrm{y}$ centro de documentación, el DEAC y el área comercial, todos ellos muy vinculados con la atención que se ofrece al visitante y la imagen que éste recibe de la institución. Los tres han sufrido y están sufriendo ciertas incidencias dada la reciente creación del museo. ${ }^{26}$

El número de visitantes que ha recibido el MUSAC en este primer año ha sido también espectacular. La primera exposición, Emergencias, fue visitada por 83.258 personas; y la segunda, con obras de Dora García, una exposi-

\footnotetext{
${ }^{26}$ El área comercial está a la espera de su puesta en marcha dado que ya han sido adjudicados a empresas comerciales y de restauración. Hasta principios de abril del año 2006, el DEAC estuvo emplazado en dicho lugar. La biblioteca también fue abierta ese mismo mes, abril de 2006, en cuyo espacio también fue otro de los lugares previos del DEAC durante la apertura del centro en abril de 2005. Definitivamente el DEAC se encuentra ya emplazado en su correspondiente ubicación dentro de las áreas de uso público.
}

ción colectiva denominada "Sujeto" y la de Shirin Neshat por $67.327 .^{27}$

La exposición que estaba en marcha durante la etapa de evaluación de público fue la tercera que tuvo lugar entre los meses de diciembre de 2005 y abril de 2006 (17 de diciembre de 2005 - 16 de abril de 2006) compuesta de las siguientes muestras:

- "Flaschengeist la caseta del alemán”, de Enrique Marty,

- "Fusion", exposición colectiva de fondos de contenido asiático u oriental que posee el museo,

- y "Pröblemäs büenös. 4 obras de Pipilotti Rist y amigäs".

Por todo lo dicho, se hace notar que el museo se enfrenta a la complejidad del comienzo de su andadura tanto en la apretada oferta de exposiciones, como en la atención a la masiva afluencia de público que asiste a ellas. Así mismo la puesta en marcha del conjunto de servicios, el aprovechamiento del espacio destinado a la exposición y de la disposición de instalaciones, debe acomodarse a esa coyuntura.

La propuesta de evaluación debía proporcionar información sobre el uso y el impacto público del museo en esos momentos y de la exposición ya en funcionamiento, es decir, una evaluación sumativa. Como tal es la que permite valorar los aspectos de la institución que están funcionando o no de cara a la interacción con el público, y sobre todo la información resultante del contacto con los visitantes.

\section{Objetivos generales, hipótesis de trabajo}

Las conversaciones mantenidas con Belén Sola, Coordinadora del DEAC del MUSAC orientaron las hipótesis de las cuales se partieron y los objetivos generales del trabajo. Así se observó esa cantidad elevada de visitantes; algunos de ellos renovaban la visita varias veces convirtiéndose en asiduos, e incluso repitiendo el uso de la visita guiada, sobre todo gente joven. Por otro lado, las características de las piezas, algunas de producción exclusiva para el museo, y la contemporaneidad de los artistas de la exposición, muchos de ellos desconocidos

\footnotetext{
${ }^{27}$ Los datos sobre el número de personas que asiste al museo han sido facilitados por el DEAC del MUSAC.
} 
para el público general, hacía que los visitantes frecuentaran el uso del servicio de visita guiada. De la misma manera, algunas piezas tenían más aceptación que otras, o eran más visitadas, por parte del público.

Por todo ello, los objetivos propuestos para evaluación se concretaron en los siguientes:

- Conocer el perfil y las características del público del museo, su opinión sobre la institución, el impacto de las colecciones y el uso de determinados servicios.

- Evaluar el montaje expositivo y el interés del público por las piezas exhibidas.

El producto de la evaluación pretendía obtener datos sobre el perfil de esos visitantes, la razón de su visita y su opinión, además del uso de determinados servicios del MUSAC. Así se podría confirmar esa hipotética afluencia masiva de público joven. De la misma manera, el análisis del montaje expositivo y el seguimiento del comportamiento del visitante validaría o no la hipótesis previa sobre la incidencia de su colocación en el interés de la pieza y daría una visión general de las preferencias, es decir de las obras más visitadas por el público.

\section{Metodología}

La metodología elegida para llevar a cabo el proyecto consistió en la elección de un sistema de trabajo que combinara técnicas tanto cualitativas como cuantitativas, que fuera útil para la consecución de resultados, y factible con los medios humanos de los que se disponía. Se desecharon otras metodología por el inconveniente fundamental de requerir gran esfuerzo de tiempo y de personal del cual se carecía.

Los tipos de evaluación que se utilizaron fueron la observación y los cuestionarios autoadministrados. La observación de los visitantes mientras realizan la visita a las diferentes exposiciones requiere un importante esfuerzo humano y tuvo que ser adaptado a las características del proyecto realizado por una única persona, sin menoscabo de la aspiración a obtener un resultado óptimo de datos. Los cuestionarios fueron más fáciles de implementar dado su diseño de carácter autoadministrado, aunque hubo que animar en determinados momentos la colaboración del visitante. Para ello se recibió la ayuda del personal de sala del museo.
Se eligió la observación para evaluar sólo el interés de las obras de las exposiciones de Enrique Marty, Fusion y Pipilotti. La técnica, aunque costosa, se consideró interesante para la obtención de datos concluyentes sobre el impacto de las piezas exhibidas y evaluación del montaje. En el caso de las dos últimas, Fusion y Pipilotti, únicamente se consideró el nivel de acceso y grado de interés de las piezas. En el caso de Enrique Marty, se evaluó también el grado de atención, tiempos de parada y secuencias de recorrido de su instalación. Se desechó la observación por cámaras ya que entorpecía la efectividad de la vigilancia y seguridad del museo.

La encuesta o cuestionario autoadministrado por los visitantes es una metodología de recopilación de información que completa las carencias o límites en el tamaño de la muestra de visitantes que se obtienen a través del anterior método. La combinación de diferentes procedimientos de obtención de datos es básica para obtener un buen perfil del visitante del museo. Por un lado aporta una definición más completa y representativa del visitante del MUSAC que el obtenido sólo a través de la observación. Por otro lado, proporciona información de primera mano sobre el impacto de las exposiciones además de la obtención de otros datos sobre su opinión, fuentes de información, motivos de visita, etc. Todo ello es de interés para el museo en su primer año de andadura. Además se consideró que el público sería receptivo a la aceptación de este tipo de metodologías de trabajo y que daba buena imagen pública de la institución al proporcionar el mensaje a los visitantes de que sus opiniones son importantes $\mathrm{y}$ tenidas en cuenta por el MUSAC para su mejora. ${ }^{28}$

Las preguntas que se realizaron fueron nueve:

- edad (de respuesta abierta),

- sexo (de respuesta cerrada, mujer/hombre),

- profesión (de respuesta cerrada: empresario, profesional liberal, funcionario, etc.),

\footnotetext{
${ }^{28}$ M. Asensio, E. Pol y M. Gomis, Ob. cit., p. 30.
} 
- si era la primera vez que visitaba el museo (de respuesta cerrada si/no),

- el número de visitas realizadas para público asiduo (de respuesta cerrada, 2, 3, 4 , etc. así hasta la opción 10 o más),

- si el visitante ha hecho uso del servicio de visita guiada (de respuesta cerrada sí/no),

- si ha hecho uso de ese mismo servicio otras veces (también de respuesta cerrada sí/no),

- cómo se enteró de las exposiciones (de respuesta cerrada, pero con la posibilidad de selección múltiple, es decir de señalar más de una opción: radio, prensa, TV, etc.),

- el motivo por el que ha asistido al museo (también de respuesta cerrada, pero con la posibilidad de selección múltiple: curiosidad y/o interés personal, interés por el museo, etc.),

- y las dos últimas de respuesta abierta sobre los aspectos del museo que hayan gustado, llamado la atención o sorprendido al visitante, y novena, final, sobre aquello que hubiera echado en falta durante la visita en el museo.

Las metodologías de trabajo elegidas fueron llevadas a cabo de manera independiente para mejor organización del trabajo y concentración de esfuerzos. Se comenzó con la observación, y la encuesta culminó el trabajo de campo.

La observación se llevó a cabo durante los meses de febrero y marzo de 2006 alternando exposiciones en diferentes horarios de mañana y tarde, con el público real entre semana y un sábado de mañana para cada exposición. Así podría valorarse la evaluación del público de fin de semana. La encuesta estuvo a disposición de los visitantes -o público real-, el último mes de la exposición, del 23 de marzo al 16 de abril, en horario de apertura del museo, de martes a domingo.

Tiempo de perma- 17 de diciembre $2005-$
nencia de las ex- 16 de abril 2006.
posiciones

\begin{tabular}{ll}
\hline $\begin{array}{l}\text { Desarrollo de la } \\
\text { observación }\end{array}$ & $\begin{array}{l}\text { Los martes, miércoles y del 14 de febrero al } \\
21 \text { de marzo y tres sába- } \\
\text { dos de marzo de 2006. }\end{array}$ \\
\hline $\begin{array}{l}\text { Tiempo de perma- } \\
\text { nencia de la en- } \\
\text { cuesta }\end{array}$ & $\begin{array}{l}23 \text { de marzo 2006-16 de } \\
\text { abril } 2006\end{array}$ \\
\hline
\end{tabular}

El proceso de toma de datos de la observación consistió en escoger aleatoriamente los visitantes que accedían a las salas para abarcar una mayor variedad en la muestra, procurando hacer las máximas anotaciones posibles, incluso dejando la posibilidad de reflejar otras cuestiones concretas en un apartado denominado "observaciones" en las fichas de toma de datos diseñadas para tal fin.

Como norma general para la anotación de dichos datos, no se tuvieron en cuenta para la evaluación los grupos de visitantes que accedían a través de visita guiada. Así, cuando dicho grupo llegaba, se paralizaba dicha evaluación, y se anulaban los datos tomados de los visitantes que se estaban observando. No cabe duda de que la presencia del grupo afecta al comportamiento de los visitantes particulares. Éstos, unas veces, se unían al mismo y, en general, dejaban de actuar con libertad.

En el caso de parejas o visitantes que acudían acompañados de varias personas se hacía una anotación de recorrido genérica para todos sus miembros, y salvo que se dispersara (aspecto que fue poco frecuente), se daba un único trayecto.

Respecto de la valoración de la muestra obtenida la situación fue diferente para ambas técnicas. La que se obtuvo de la observación fue de: 64 personas para Enrique Marty, 77 para Fusion y 144 para Pipilotti Rist. En esta última se pudo evaluar un mayor número de personas en comparación con Marty o Fusion. La afluencia de público, fluida pero espaciada, y la disposición cómoda de los montajes en las diferentes salas de la exposición de Pipilotti Rist, permitía un fácil seguimiento de esos aspectos. En total se tomó nota de 285 visitantes durante la observación.

Si para la observación no se fijó ningún mínimo ni máximo de personas para realizar la evaluación, en la encuesta sí se determinó un número mínimo para una mayor fiabilidad de los resultados. Ésta se calculó en función de los 
datos existentes sobre público del museo. Se valoró el número que se necesitaría para que los resultados fueran concluyentes.

La muestra que se tomó se calculó en función de los datos existentes sobre público en el MUSAC. Hasta la fecha en la cual se empezó a trabajar sobre la encuesta habían pasado 23.486 personas por esta tercera exposición. Por otro lado, aún no había cumplido un año de la inauguración del museo, por lo tanto no había datos sobre las visitas que había recibido el mismo en los meses de marzo y abril de temporadas anteriores, sólo los datos de la segunda quincena de abril de 2005. Esta información no era útil por ser demasiado elevada debido a la afluencia masiva por la inauguración. Por lo tanto, sólo se disponía de la cifra de visitantes acumulados desde la inauguración de esta tercera muestra en diciembre de 2005, esos 23.486 visitantes, y la cifra de 6.005 visitas que había recibido en el mes de febrero de 2006. Aplicando los cálculos necesarios se estimó que se necesitarían unas 668 encuestas pasadas de forma aleatoria con un nivel de confianza del $95,5 \%$ y un error muestral del $3 \%$, para que los resultados fueran concluyentes.

Así se distribuyeron 1.500 cuestionarios, de los cuales, al final 741 fueron rellenados por el público, 436 sobraron y el resto, 323, no fueron entregadas. Como la cifra de encuestas superó en 73 la cifra estimada, el error muestral final quedó en 2,9\%. El nivel de significatividad es muy aceptable dado que se desconocía cuánta gente iba a visitar el museo durante la etapa de trabajo de campo.

\begin{tabular}{|c|c|}
\hline $\begin{array}{l}\text { Población total } \\
\text { final: }\end{array}$ & $\begin{array}{l}14.195 \text { visitantes (febrero- } \\
\text { marzo) }\end{array}$ \\
\hline Muestra: & 741 encuestados \\
\hline Nivel de error: & $\begin{array}{l} \pm 2,9 \% \text { a un nivel de confian- } \\
\text { za del } 95,5 \%\end{array}$ \\
\hline
\end{tabular}

Respecto a la evaluación según el día de la semana o la hora del día se obtuvieron ciertas ideas en relación a la participación del público en el rellenado de cuestionarios y en la observación en sala. En el caso de la encuesta se observó que el público participaba de manera muy irregular tanto entre semana como los fines de semana, sin embargo, tras la animación que se puso en marcha la segunda semana, pasando encuestas personalmente al visitante y entregándole un obsequio, dicho público se implicó más.

\section{Evaluación de resultados}

El MUSAC ha recibido, desde su inauguración hasta la fecha de 16 de abril, final de la evaluación, 249.519 visitantes, durante lo que se puede considerar el primer año de apertura. No cabe duda de que es una cifra muy elevada de público asistente si se compara con las cifras de visitantes de otros museos. ${ }^{29}$ La gratuidad de la entrada es un factor a tener en cuenta para entender esta circunstancia.

De esos 249.519 visitantes, a la exposición inaugural, "Emergencias", acudieron 83.258 personas; y la segunda, con obras de Dora García, la exposición colectiva denominada "Sujeto" y la de Shirin Neshat fueron 67.327 visitantes, como ya ase apuntó. Y por último, a la exposición sometida a evaluación, con la instalación de Enrique Marty, la colectiva "Fusion" y las obras de Pipilotti Rist, asistieron 98.934 personas.

En comparación, ésta última muestra ha sido la que mayor cifra de visitantes ha recibido de las tres, incluso más que la inaugural que estuvo más tiempo y suele ser la que más público atrae a causa de la expectación de la apertura de cualquier museo. ${ }^{30}$

El público que asistió al MUSAC fue mayoritariamente joven, y por lo tanto, en su mayoría, estudiantes. Este resultado confirmó la hipótesis previa que argumentaba esa singularidad. Esta presencia mayoritaria de público joven se ve motivada por el programa de actividades para el público escolar y de institutos que tiene diseñado el DEAC.

Respecto de la proporción de público femenino con respecto al masculino, es mayor

\footnotetext{
${ }^{29}$ Estadística de museos y colecciones museográficas, (2005) Ministerio de Cultura, Madrid: 85 y ss.

${ }^{30} \mathrm{Si}$ se compara con las cifras de otros museos nacionales es esos tres meses (de enero a marzo) de funcionamiento también son espectaculares: por ejemplo el Museo Arqueológico Nacional ha recibido 73.594. Fuente:

http://www.mcu.es/jsp/plantilla wai.jsp?id $=633 \&$ conte nido $=/$ museos $/ \mathrm{col} / \mathrm{dif} /$ avance.jsp $\&$ anno $=2006 \& \mathrm{mes}=$ 04\&area $=$ museos

A la fecha de cierre de este documento está en marcha la cuarta exposición del museo y las cifras siguen manteniendo el ritmo de asistencia de visitantes, más elevado dado que está teniendo lugar en época estival. La cifra de visitantes va en constante y progresivo aumento.
} 
en el caso de mujeres. Si embargo la diferencia entre ambas no fue demasiado acusada.

Durante la observación de los visitantes se obtuvo que las piezas que más captaron la atención fueron la obra de Do-Ho Shu, Corridor, dentro de la exposición colectiva Fusion, Homo Sapiens sapiens de Pipilotti Rist, y el tiovivo y las casetas de la instalación de Marty. En cambio, las que menos llamaron su atención fueron las obras que estaban emplazadas en lugares poco visibles o de accesibilidad poco clara, como por ejemplo Dialoge with my self de Morimura, tal y como se planteó en una de las hipótesis previas de las que partió la evaluación.

A través de la encuesta se observó que aún existe una cantidad elevada de público que vista por primera vez el museo. Aunque el número de visitantes es importante, la cantera de público potencial que aún no ha acudido al museo es considerable dada la reciente apertura del mismo.

De los visitantes que habían acudido al museo más de una vez, la mayoría lo hicieron en su segunda ocasión, decreciendo en proporción los visitantes que frecuentan más su visita.
La proporción de los visitantes encuestados que hicieron uso del servicio de visita guiada del museo fue relativamente baja con respecto al resultado que se había previsto. No obstante es uno de los servicios más demandados del museo según las memorias del DEAC.

La principal fuente de información sobre el MUSAC a la que recurrieron los visitantes encuestados fue a través de un conocido, seguido de la prensa. Esto demuestra que la campaña de marketing y medios que lleva el Departamento de Comunicación obtiene resultados.

El motivo de visita mayoritario por el cual el público ha asistido al museo es por curiosidad y/o interés personal. De nuevo, la novedad de la institución museística es uno de los factores que atraen al público en su visita.

Y para concluir, respecto a la opinión, al público le ha gustado el museo en general, tanto en sus exposiciones, como por el edificio o por el servicio y atención recibida, mostrándose en su mayoría satisfecho. Sin embargo, hay sectores que han echado en falta algún tipo concreto de obras o una sala de exposición permanente con fondos de la colección estable del museo.

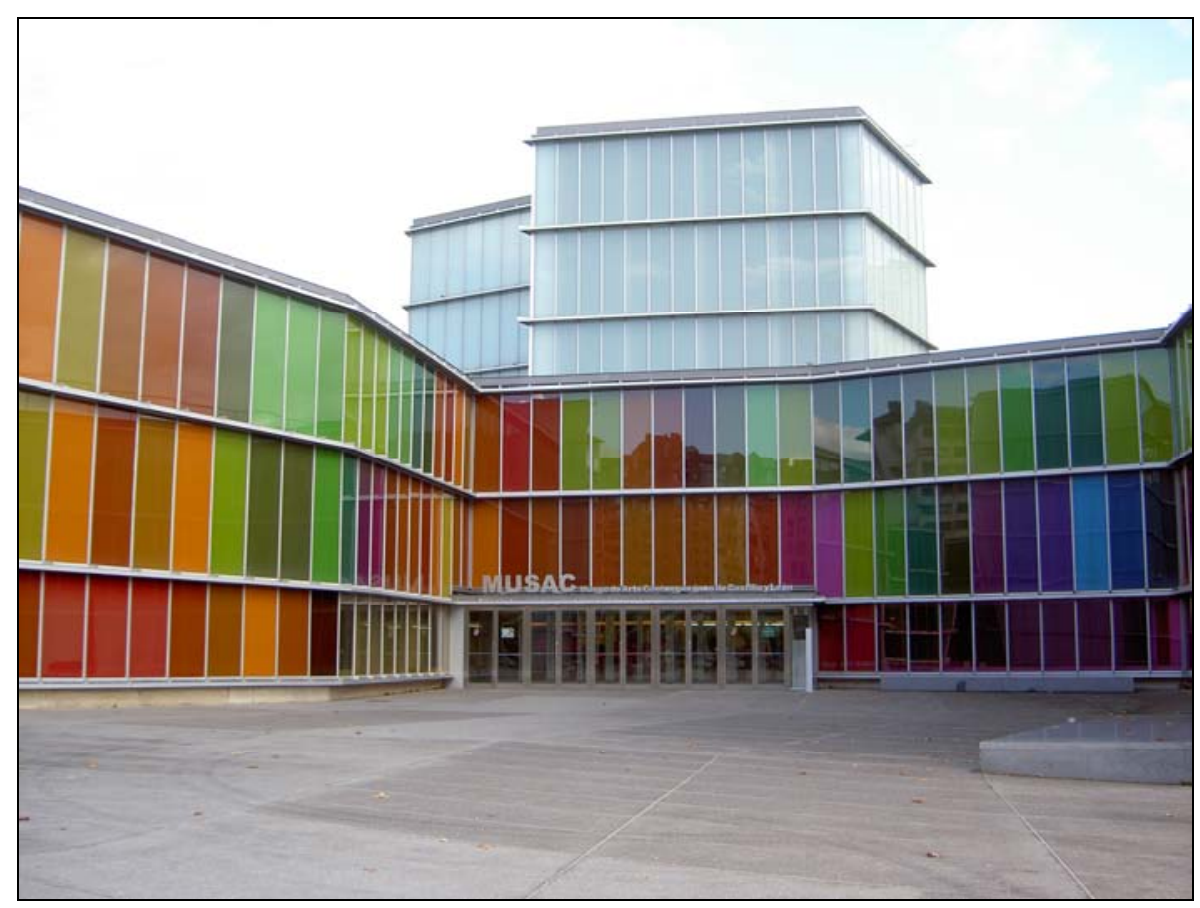

- Fig. 1. Vista exterior del museo. Fotografía de la autora. 


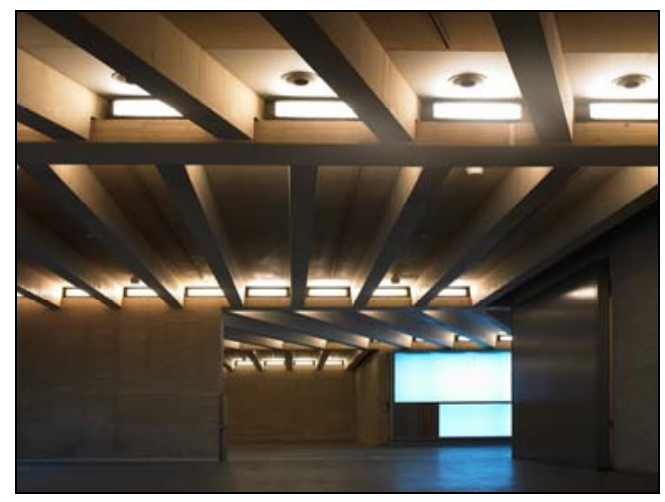

- Fig. 2. Vista interior de las salas de exposición. MUSAC, (C) Ángel Marcos.

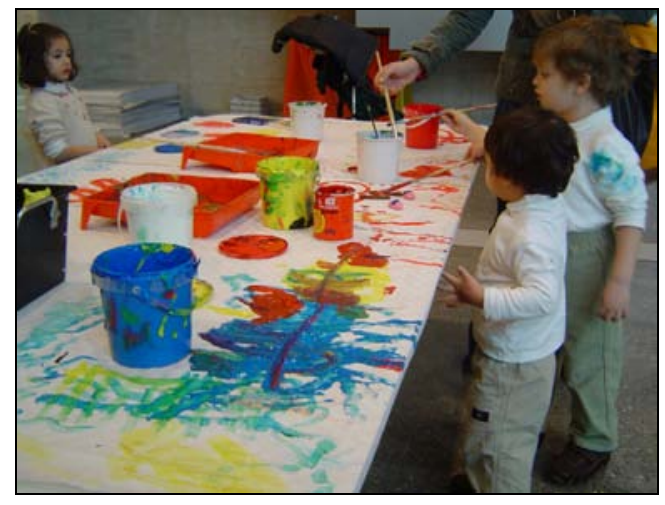

- Fig. 4. Taller infantil. D.E.A.C. MUSAC.

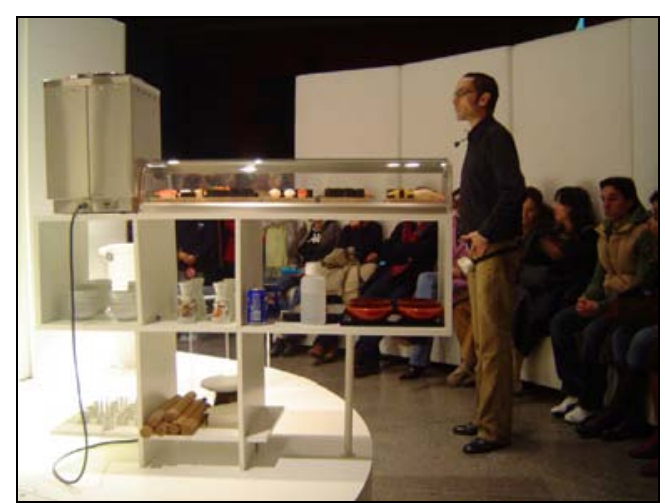

- Fig. 3. Visita guiada de las exposición "Fusion: aspectos de la cultura asiática en la colección MUSAC". D.E.A.C. MUSAC.

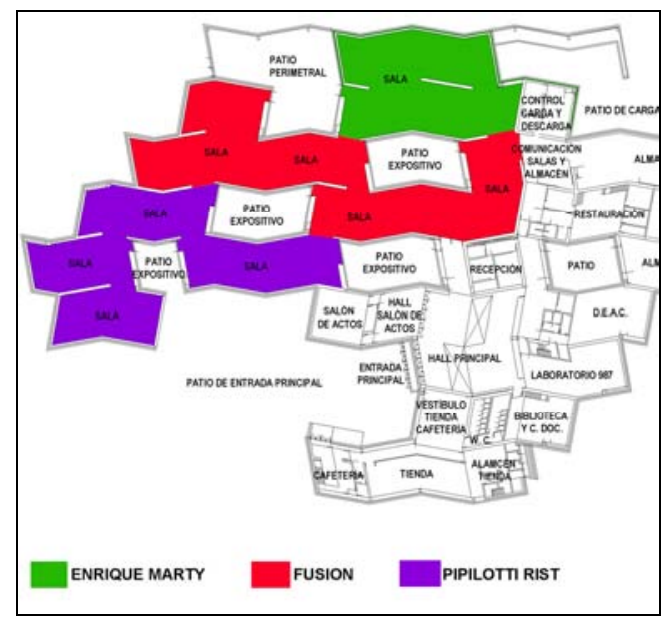

- Fig. 5. Plano del museo con la leyenda de las exposiciones evaluadas. Elaboración propia. 